\title{
Coregulation of ionic currents maintaining the duty cycle of bursting
}

\author{
William Barnett ${ }^{1 *}$, Martin Anquez ${ }^{2}$, Gennady Cymbalyuk $k^{2,3}$ \\ From Nineteenth Annual Computational Neuroscience Meeting: CNS*2010 \\ San Antonio, TX, USA. 24-30 July 2010
}

Central pattern generators (CPGs) are the oscillatory neuronal networks which control rhythmic movements of animals. Some CPGs keep the phase relationships between the neurons' oscillatory activities over a wide range of the cycle periods. Maintenance of the duty cycle of the bursting activity could be a key feature for a variety of dynamical mechanisms supporting phase constancy in oscillatory neuronal networks. It is a form of cellular homeostasis of neuronal activity. Among other currents, hyperpolarization-activated currents and potassium currents have been shown to be a ubiquitous target for modulation and homeostasis [1,2].

Here we present a novel mechanism of coregulation of currents which preserves duty cycle of bursting activity over a range of cycle periods. We develop a generic lowdimensional Hodgkin-Huxley type model stemming from a model of the leech heart interneuron under certain pharmacological conditions [3]. Application of $\mathrm{Co}^{2+}$ and 4-AP blocks $\mathrm{Ca}^{2+}$ currents, the synaptic currents and most of the $\mathrm{K}^{+}$currents. The model contains the slow potassium current $\left(\mathrm{I}_{\mathrm{K} 2}\right)$, the fast sodium current $\left(\mathrm{I}_{\mathrm{Na}}\right)$. Our new model also includes the hyperpolarization activated current $\left(\mathrm{I}_{\mathrm{h}}\right)$. Bifurcation theory allows us to make predictions concerning the temporal characteristics of the dynamics of bursting nearby the critical transitions between activities.

Shilnikov \& Cymblayuk showed that the transition from bursting into tonic spiking (blue sky catastrophe) determines the dependence of the burst duration on the voltage of half-activation of $\mathrm{I}_{\mathrm{K} 2}\left(\theta_{\mathrm{K} 2}\right)$ as one over square root of the parameter value [4]. Here we show that the half-activation potential of $I_{h}\left(\theta_{h}\right)$ controls the interburst interval as one over square root of the parameter value. We investigate the activity of the model to identify mechanisms of coregulation of $\mathrm{I}_{\mathrm{K} 2}$ and $\mathrm{I}_{\mathrm{h}}$ maintaining the

\footnotetext{
* Correspondence: gcymbalyuk@gsu.edu

${ }^{1}$ Department of Biology, Georgia State University, Atlanta, Georgia 30303, USA
}

duty cycle. Bifurcation analysis of the model was performed using $\theta_{K 2}$ and $\theta_{h}$, as controlling parameters. We investigated the temporal characteristics of bursting activity. We identified a saddle node bifurcation for periodic orbits determining the blue sky catastrophe [4] and a saddle node bifurcation for stationary states (SNIC) [5]. We showed the temporal characteristics of bursting depend on the location of the bifurcation curves. By coordinating the two parameters, we were able to increase the period such that the burst duration and interburst interval maintained constant proportion. The coregulation consists of a negative correlation of $\theta_{\mathrm{K} 2}$ and $\theta_{h}$, which is steeper for the higher duty cycles.

\section{Acknowledgements \\ Supported by NSF PHY-0750456.}

\section{Author details}

${ }^{1}$ Department of Biology, Georgia State University, Atlanta, Georgia 30303, USA. ${ }^{2}$ Department of Physics and Astronomy, Georgia State University, Atlanta, Georgia 30303, USA. ${ }^{3}$ Neuroscience Institute, Georgia State University, Atlanta, Georgia 30303, USA.

\section{Published: 20 July 2010}

\section{References}

1. MacLean JN, Zhang Y, Johnson BR, Harris Warrick RM: Activity-independent homeostasis in rhythmically active neurons. Neuron 2003, 37(1):109-120.

2. MacLean JN, Zhang Y, Goeritz ML, Casey R, Oliva R, Guckenheimer J, HarrisWarrick RM: Activity-independent coregulation of IA and Ih in rhythmically active neurons. J Neurophysiol 2005, 94(5):3601-3617.

3. Cymbalyuk GS, Calabrese RL: A model of slow plateau-like oscillations based upon the fast $\mathrm{Na}+$ current in a window mode. Neurocomputing 2001, 38:159-166.

4. Shilnikov A, Cymbalyuk G: Transition between tonic spiking and bursting in a neuron model via the blue-sky catastrophe. Phys Rev Lett 2005, 94(4):048101.

5. Ermentrout B: Type 1 membranes, phase resetting curves, and synchrony. Neural Comput 1996, 8(5):979-1001.

doi:10.1186/1471-2202-11-S1-O3

Cite this article as: Barnett et al: Coregulation of ionic currents maintaining the duty cycle of bursting. BMC Neuroscience 201011 (Suppl 1):O3. 